\title{
Calculation of the phonon density of states and related thermodynamic properties for
} trigonal selenium

\author{
Hansen, Flemming Yssing; Alldredge, G. P.; McMurry, H. L.
}

Published in:

Journal of Chemical Physics

Link to article, DOI:

$10.1063 / 1.444795$

Publication date:

1983

Document Version

Publisher's PDF, also known as Version of record

Link back to DTU Orbit

Citation (APA):

Hansen, F. Y., Alldredge, G. P., \& McMurry, H. L. (1983). Calculation of the phonon density of states and related thermodynamic properties for trigonal selenium. Journal of Chemical Physics, 78(2), 921-924.

https://doi.org/10.1063/1.444795

\section{General rights}

Copyright and moral rights for the publications made accessible in the public portal are retained by the authors and/or other copyright owners and it is a condition of accessing publications that users recognise and abide by the legal requirements associated with these rights.

- Users may download and print one copy of any publication from the public portal for the purpose of private study or research.

- You may not further distribute the material or use it for any profit-making activity or commercial gain

- You may freely distribute the URL identifying the publication in the public portal 


\title{
Calculation of the phonon density of states and related thermodynamic properties for trigonal selenium
}

\author{
F. Y. Hansen \\ Fysisk-Kemisk Institut, The Technical University of Denmark, DTH 206, DK 2800 Lyngby, Denmark
}

G. P. Alldredge

Department of Physics, University of Missouri, Columbia, Missouri 65211

H. L. McMurry

Department of Physics, Idaho State University, Pocatello, Idaho 83209

(Received 14 September 1981; accepted 4 February 1982)

\begin{abstract}
The phonon density of states for trigonal selenium has been calculated on the basis of a short range force model giving good overall agreement with experimental room temperature phonon dispersion data. A qualitative comparison with an experimental determination of the phonon density of states shows similarities in the gross features, but the experimental data lacks many of the finer details shown by the theoretical results due to resolution effects. The lattice dynamical contribution to the heat capacity $C_{V}$ is calculated and is found to be in good agreement with experimental determinations of $C_{p}$ after transforming $C_{p}$ to $C_{v}$.
\end{abstract}

\section{INTRODUCTION}

In this paper, we report the results of a calculation of the phonon density of states for trigonal selenium and the heat capacity $C_{V}$. The calculations are based on a short range force model for trigonal selenium suggested by us. ${ }^{1}$ With the neutron scattering measurements ${ }^{2}$ of the phonon dispersion relations in various symmetry directions, there is a good basis for a thorough test of force models. The experimental data showed that the simple models based on spectroscopic data and elastic moduli were inadequate. More refined models gave a better agreement with the neutron data, in particular for the lower frequencies. All models, however, showed almost flat optic bands, whereas the neutron data showed a considerable dispersion of those frequencies. The introduction of long range electrostatic forces did not improve the agreement noteworthy.

In our model, the force field is defined in terms of symmetrized coordinates, which partly reflect the symmetry of the space group. The intrachain forcefield was projected from a valence type field including a bond stretch, angle bend, and dihedral torsion. With this field, we obtained the appropriate dispersion of the upper optic modes. The interchain force field was projected from relative rotations and translations of groups of atoms in adjacent chains. In all, it was found that our model gave a better overall agreement with experimental data than other models. It is, therefore, expected that a calculation based on this model would give a reliable phonon density of states for trigonal selenium.

The phonon density of state has been determined experimentally, using coherent inelastic neutron scattering from powdered samples. ${ }^{3}$ It is obvious that the calculated density of state shows more features than the experimental results, since the latter has been resolution broadened. Standard thermodynamic relations have been used to calculate the lattice dynamical contribution to the heat capacity $C_{V}$ from the density of states, and the results are compared with experimental results. ${ }^{4,5}$ Although the agreement is good, it is remembered that the thermodynamic functions are not very sensitive to details in the frequency distribution, due to the averaging.

\section{Calculations}

The phonon density of states has been calculated using the linear analytic extrapolation method developed by Raubenheimer and Gilat, ${ }^{6}$ an accurate and rapid method for calculating the frequency distribution. Briefly, this method consists of solving the secular equation associated with the phonon dynamical matrix at a relatively small number of mesh points (here 1287) in the irreducible section of the first Brillouin zone and then, by means of a linear analytic extrapolation, extracting all the phonon frequencies within small volumes centered at the mesh points. The shape of these volumes is immaterial, as long as they fill the irreducible Brillouin zone properly, i.e., without overlaps or gaps. This means that the mesh of points chosen need not be uniform. In the present case, where the Brillouin zone has hexagonal symmetry, the irreducible zone is filled exactly with rectangular and triangular prisms. The irreducible zone has been subdivided into three regions, with an increasing density of mesh points going from the zone boundary towards the center, in order to increase the accuracy. We have used the RaubenheimerGilat program for hexagonal systems ${ }^{8}$ and replaced the subroutines concerned with the generation of the $d y$ namical matrix with our routines. The results are shown in Fig. 1, which is a histogram representation of the frequency distribution with a channel width of $0.01 \mathrm{THz}$.

\section{EXPERIMENTAL DATA}

A direct comparison between the calculated density of states in Fig. 1 and the experimental results is not possible, as already mentioned, before the former has 


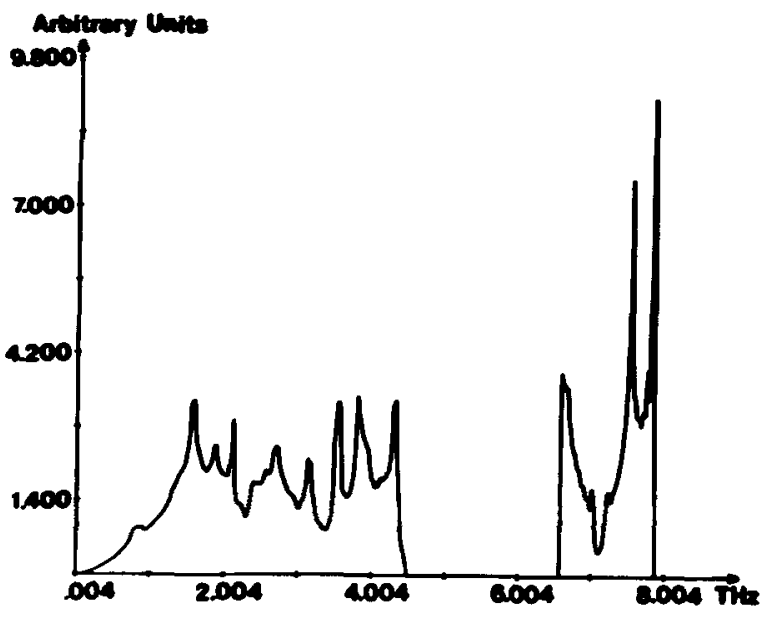

FIG. 1. Calculated phonon density of states for trigonal selenium.

been resolution broadened with the resolution function in the time-of-flight neutron measurements.

The thermodynamic data are taken from Gross et al. , " where the heat capacity $C_{p}$ in the temperature range $10-300 \mathrm{~K}$ is compiled from other authors and where measurements of the linear thermal expansion coefficients $\alpha_{\| 1}$ and $\alpha_{1}$ as a function of temperature in the same temperature range are reported. $\alpha_{11}$ and $\alpha_{1}$ are the coefficients of linear thermal expansion in the direction of the chains and perpendicular to the chains, respectively. Together with adiabatic elastic moduli $C_{i k}$ determined by Meissner and Mimkes, ${ }^{5}$ it is possible to convert the experimental heat capacity $C_{p}$ to $C_{V}$ for comparison with the calculated heat capacity.

The standard thermodynamic relation between $C_{p}$ and $C_{V}$ may be written

$$
C_{p}=C_{V}\left(1+\alpha^{2} K_{S} V T / C_{p}\right),
$$

where $\alpha=1 / V(\partial V / \partial T)_{\phi}$ is the isobaric thermal expansion coefficient and $K_{S}=-V(\partial p / \partial V)_{T} C_{p} / C_{V}$ is the adiabatic modulus.

In order to apply Eq. (III 1), we need relations between

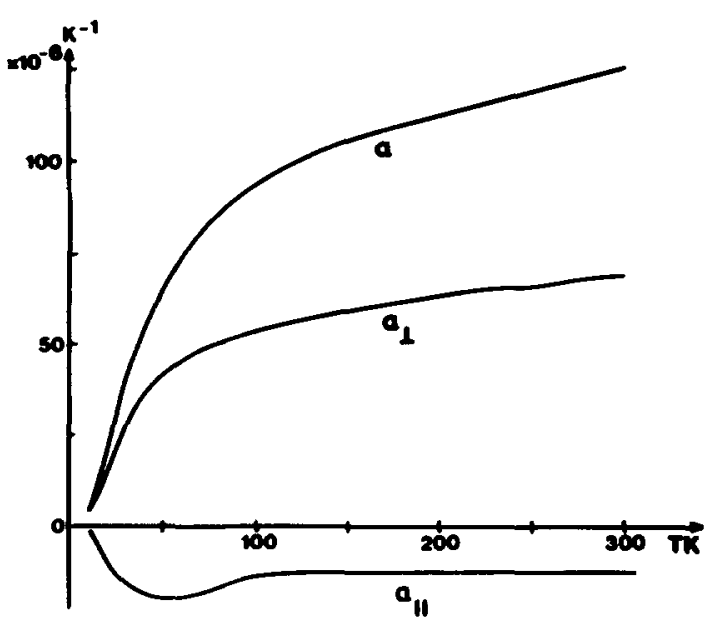

FIG. 2, The linear thermal expansion coefficients $\alpha_{11}, \alpha_{1}$, and the thermal expansion coefficient $\alpha$ for trigonal selenium.

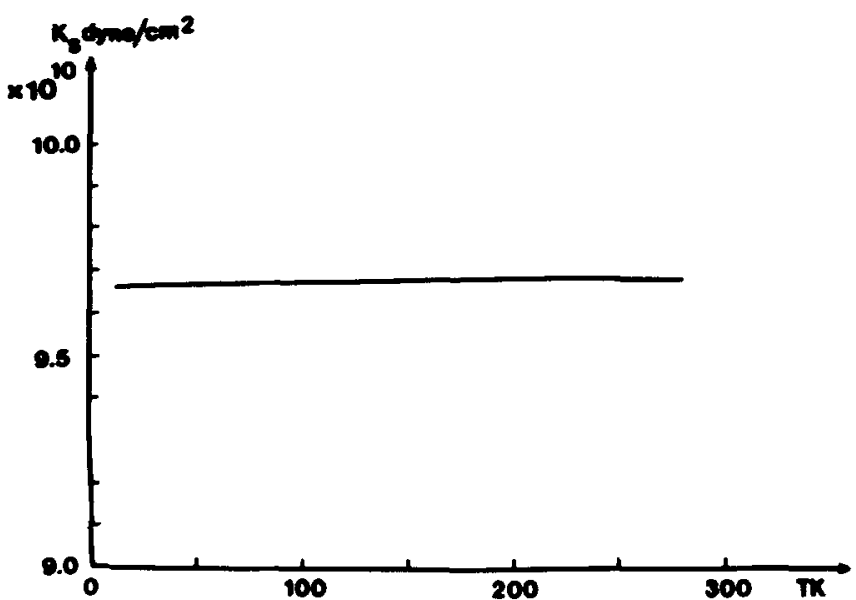

FIG. 3. The adiabatic bulk modulus for trigonal selenium calculated from adiabatic elastic moduli.

the linear thermal expansion coefficients $1 / l(\partial l / \partial T)_{p}$ and $\alpha$, and the adiabatic elastic moduli $C_{i k}$ and $K_{S}$. Consider a small rectangular prism with dimensions $l_{x}, l_{y}, l_{x}$, then

$d V=\left(\frac{\partial V}{\partial l_{x}}\right)_{l_{y} l_{z}} d l_{x}+\left(\frac{\partial V}{\partial l_{y}}\right)_{l_{x^{l}}} d l_{y}+\left(\frac{\partial V}{\partial l_{z}}\right)_{l_{x^{\prime} y}} d l_{x}$,

or

$\left(\frac{\partial V}{\partial T}\right)_{p}=\left(\frac{\partial V}{\partial l_{x}}\right)_{l_{y} l_{x}}\left(\frac{\partial l_{x}}{\partial T}\right)_{p}+\left(\frac{\partial V}{\partial l_{y}}\right)_{l_{x} l_{x}}\left(\frac{\partial l_{y}}{\partial T}\right)_{p}+\left(\frac{\partial V}{\partial l_{z}}\right)_{l_{x} l_{y}}\left(\frac{\partial l_{x}}{\partial T}\right)_{D}$,

and if we assume the chain axis in the $z$ direction,

$\alpha=\frac{1}{V}\left(\frac{\partial V}{\partial T}\right)_{p}=\alpha_{x}+\alpha_{y}+\alpha_{z}=2 \alpha_{1}+\alpha_{11}$,

which is the desired relation for $\alpha, \alpha, \alpha_{1}$ and $\alpha_{11}$ are shown in Fig. 2 .

In order to evaluate an expression for the bulk modulus of the crystal, we start with the stress-strain relation for a trigonal crystal, which has the for ${ }^{7}$

$\left[\begin{array}{c}X_{x} \\ Y_{y} \\ Z_{z} \\ Y_{z} \\ Z_{x} \\ X_{y}\end{array}\right]\left[\begin{array}{cccccc}C_{11} & C_{12} & C_{13} & C_{14} & 0 & 0 \\ C_{12} & C_{11} & C_{13} & -C_{14} & 0 & 0 \\ C_{13} & C_{13} & C_{33} & 0 & 0 & 0 \\ C_{14} & -C_{14} & 0 & C_{44} & 0 & 0 \\ 0 & 0 & 0 & 0 & C_{44} & C_{14} \\ 0 & 0 & 0 & 0 & C_{14} & \frac{1}{2}\left(C_{11}-C_{12}\right)\end{array}\right]\left[\begin{array}{l}l_{x x} \\ l_{y y} \\ l_{z x} \\ l_{y z} \\ l_{x x} \\ l_{x y}\end{array}\right]$.

(III 3)

Here $X_{x} \cdots X_{y}$ are the stresses, $C_{i k}$ the elastic moduli, and $l_{x x} \cdots l_{x y}$ the strains. The bulk modulus may be written

$$
K=-V(p / \Delta V),
$$

where $\Delta V$ is the dilation caused by the isotropic pressure $p$. The state of isotropic pressure $p$ corresponds to stresses $X_{x}=Y_{y}=Z_{z}=-p$, with the remaining stress components equal to zero. Furthermore, $\Delta V / V=l_{\mathbf{x x}}$ $+l_{y y}+l_{z x}$. We therefore have to calculate $l_{x x}, l_{y y}$, and $l_{k z}$ in terms of the stress components and since the $C_{t k}$ are the adiabatic elastic moduli, we find 


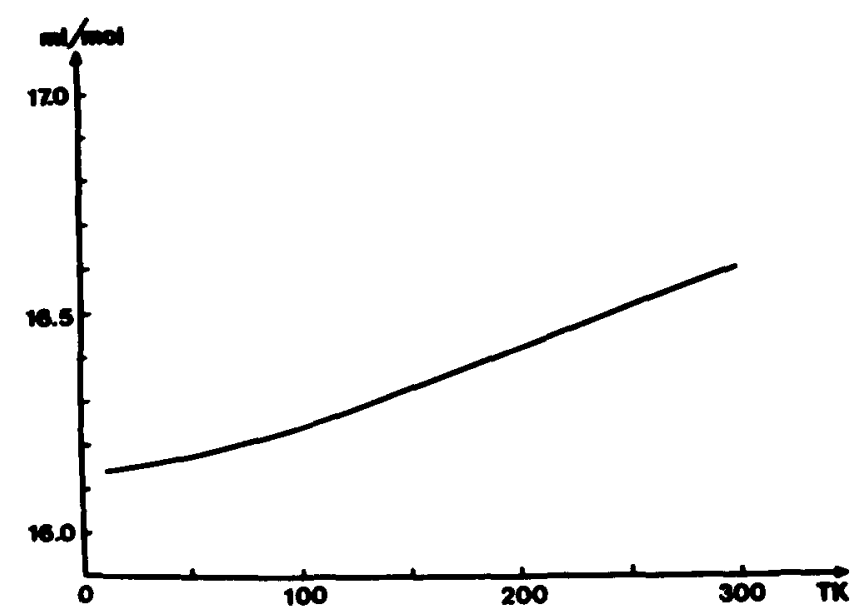

FIG. 4. The molar volume of trigonal selenium as a function of temperature, based on the thermal expansion coefficient.

$K_{S}=-\frac{p}{l_{x x}+l_{y y}+l_{z \varepsilon}}=\frac{\left(C_{11}+C_{12}\right) C_{33}-2 C_{13}^{2}}{\left(C_{11}+C_{12}\right)+2 C_{33}-4 C_{13}}$

and the results are shown in Fig. 3. The molar volume $V$ is calculated as a function of temperature from $\alpha$ and shown in Fig. 4.

The heat capacities are shown in Fig. 5 and in Table I.

\section{RESULTS AND DISCUSSION}

The calculated phonon density of states in Fig. 1 has a very complex structure reflecting a large number of critical points, as expected from the phonon dispersion relations. The experimental results have a simpler structure and quantitative comparison with our results is not possible until the latter has been resolution broadened. Qualitatively they show the same gross

TABLE I. Experimental heat capacities $C_{p}(\operatorname{expt})$ $C_{V}$ (expt) and calculated heat capacities $C_{V}$.

\begin{tabular}{|c|c|c|c|}
\hline$T(\mathrm{~K})$ & $C_{p}(\exp ) / R$ & $C_{V}(\exp ) / R$ & $C_{V} / R$ \\
\hline 10 & $\{0.0629$ & $0.0629\}$ & 0.0721 \\
\hline \multirow[b]{2}{*}{15} & 0.189 & 0.189 & \multirow[b]{2}{*}{0.193} \\
\hline & $\left\{\begin{array}{l}0.143 \\
\text {. }\end{array}\right.$ & $0.143\}$ & \\
\hline \multirow{2}{*}{20} & $\{0.337$ & $0.377\}$ & \multirow{2}{*}{0.349} \\
\hline & 0.332 & $0.322\}$ & \\
\hline \multirow{2}{*}{25} & $\{0.574$ & $0.574\}$ & \multirow{2}{*}{0.519} \\
\hline & 0.554 & $0.554\}$ & \\
\hline 30 & 0.765 & 0.765 & 0.683 \\
\hline 35 & 0.916 & 0.916 & 0.842 \\
\hline 40 & 1.06 & 1.06 & 0.989 \\
\hline 45 & 1.19 & 1.19 & 1.13 \\
\hline 50 & 1.31 & 1.31 & 1.25 \\
\hline 55 & 1.44 & 1.43 & 1.37 \\
\hline 60 & 1.54 & 1.53 & 1.48 \\
\hline 70 & 1.74 & 1.73 & 1.68 \\
\hline 80 & 1.91 & 1.90 & 1.85 \\
\hline 90 & 2.05 & 2.04 & 2.00 \\
\hline 100 & 2.18 & 1.16 & 2.12 \\
\hline 130 & 2.47 & 2.44 & 2.40 \\
\hline 160 & 2.65 & 2.61 & 2.57 \\
\hline 190 & 2.77 & 2.73 & 2.68 \\
\hline 245 & 2.91 & 2.85 & 2.80 \\
\hline 300 & 3. 01 & 2.92 & 2.86 \\
\hline
\end{tabular}

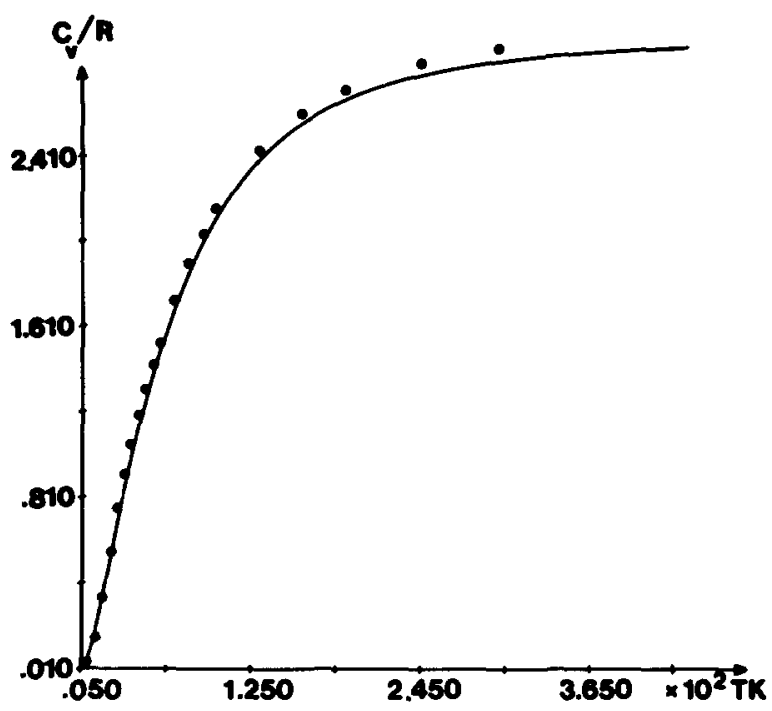

FIG. 5. The lattice dynamical contribution to the heat capacity $C_{V}$ of trigonal selenium. The dots are experimental data derived from data in Ref. 4.

features. The experimental data show three peaks in the lower frequency band at 1.6, 2.7, and $3.9 \mathrm{THz}$, respectively. This is qualitatively in agreement with our results, in particular, after they have been resolution broadened. Note, however, that the intensity of the first peak in the experimental data is larger than the intensity of the other peaks in that band, whereas, our data indicate that the intensity of the peaks is about the same.

The gap between the lower frequency region and the high frequency region extends from 4.6-6.2 THz in the experimental results as compared to our results $4.5-$ 6. $6 \mathrm{THz}$. In the high frequency region, the experi mental peak position is at 7.2 THz, whereas it is estimated that the peak position in the resolution broadened density of states in Fig. 1 is at about 7.5 THz.

The agreement between the experimental and calculated heat capacities is good, as seen from Table I and Fig. 5, taking the experimental uncertainties in particular for the elastic moduli and their temperature dependence into account. In Fig. 6 is shown the Debye

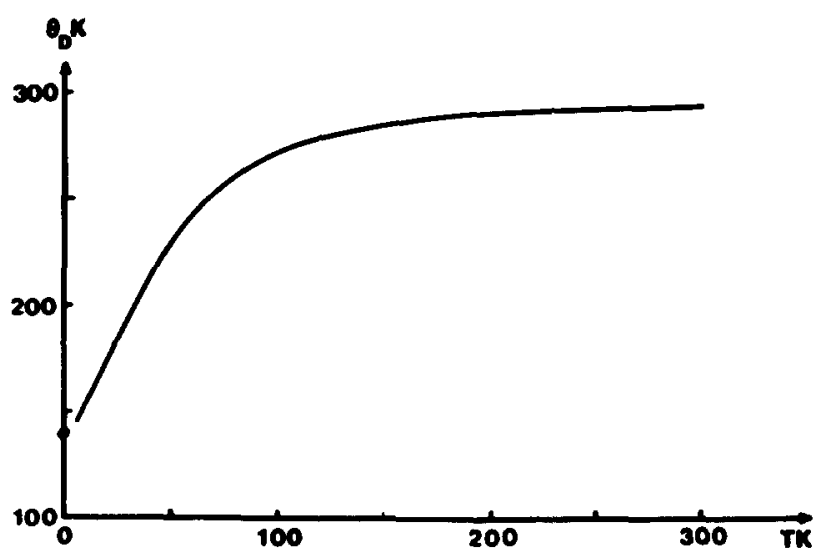

FIG. 6. The Debye temperature $\theta_{D}$ of trigonal selenium as a function of temperature, determined from the calculated heat capacities $C_{\boldsymbol{v}}$. 
temperature of the crystal determined from the calculated heat capacities. The low temperature Debye temperature $\sim 150 \mathrm{~K}$ is in agreement with most low temper ature heat capacity measurements. (The room temperature elastic constants of Mort $^{8}$ yield $\theta_{D}=138.4 \mathrm{~K}$ at 0 K.) Yet, recently, Meissner and Mimkes ${ }^{5}$ have reported the much higher low temperature Debye temperature of $186 \mathrm{~K}$ obtained from measurements on a high quality single crystal grown from a thallium-doped melt. This high value is explained in a more stiffened lattice for the perfect crystal, whereas, smaller Debye temperatures result from a softening of the polycrystal, due to microscopic disorder. Assuming that the low temperature heat capacity is dominated by the elastic properties of the solid, they calculated the Debye temperature of 171 K. They speculate that a piezoelectric stiffening may bring the Debye temperature up to the one determined by heat capacity measurements. Our results are based on room temperature dispersion relations and thus room temperature elastic moduli leading to a Debye temperature of $150 \mathrm{~K}$.

\section{ACKNOWLEDGMENTS}

This work was supported in part by NATO Grant No 。
1710 and the Danish Natural Science Council. We thank R. M. Nicklow for supplying us a copy of the Raubenheimer Gilat program for hexagonal systems.

${ }^{1}$ F. Y. Hansen and H. L. McMurry, J. Chem. Phys. 72, 5550 (1980).

${ }^{2}$ W. D. Teuchert, R. Geick, G. Landwehr, H. Wendel, and W. Weber, J. Phys. C 8, 3725 (1975); W. C. Hamilton, B. Lassier, and M. I. Kay, J. Phys. Chem. Solids 35, 1089 (1974).

${ }^{3} \mathrm{~F}$. Gompf, in The Physics of Selenium and Tellurium, Springer Series in Solid-State Sciences, edited by E. Gerlach and

P. Grosse (Springer, Berlin, 1980), Vol. 13, pp. 64-67.

${ }^{4}$ R. Grosse, P. Krause, M. Meissner, and A. Tausend, J. Phys. C 11, 45 (1978).

${ }^{5} \mathrm{M}$. Meissner and J. Mimkes, in The Physics of Selenium and Tellurium, Springer Series in Solid-State Sciences, edited by E. Gerlach and P. Grosse (Springer, Berlin, 1980), pp. 7480 .

${ }^{6}$ G. Gilat and L. J. Raubenheimer, Phys. Rev. 144, 390 (1966); L. J. Raubenheimer and G. Gilat, ibid. 157, 586 (1967).

${ }^{7} \mathrm{~F}$. I. Fedorov, Theory of Elastic Waves in Crystals (Plenum, New York, 1968), p. 31.

${ }^{8}$ J. Mort, J. Appl. Phys. 38, 1314 (1967). 